\title{
A LOW COST COARSE/FINE \\ PIEZOELECTRICALLY ACTUATED \\ MICROGRIPPER WITH FORCE \\ MEASUREMENT ADAPTED TO EUPASS \\ CONTROL STRUCTURE.
}

\author{
Kanty Rabenorosoa, Yassine Haddab, Philippe Lutz. \\ Laboratoire d'Automatique de Besançon \\ LAB UMR CNRS 6596 \\ 24, rue Alain Savary \\ 25000 BESANCON \\ France
}

\begin{abstract}
Over the last few years the demand for miniature objects and devices has continuously increased. So the need for MEMS (Micro Electro Mechanical Systems) and MOEMS (Micro Opto Electro Mechanical Systems) in various fields has become more and more important. New micromanipulation technologies must be developed that take into account the specificities of MEMS and MOEMS. For performing manipulation tasks, gripping is one of the fundamental functions. In this paper, the design and the control of a microgripper dedicated to very high precision tasks are presented. The LAB (Laboratoire d'Automatique de Besançon) has developed a microgripper based on the piezoelectric properties [1]. This gripper is equipped with a precise sensor for force measurement. Force measurement is very important in order to avoid damaging or destroying the fragile object. Various approaches were used to model and calibrate the microgripper. The control of the gripper is done using the EUPASS Control Structure [6].
\end{abstract}

\section{Structure of the system}

To handle micro-objects in a wide range of sizes, the designed microgripper is made up of two different parts (see Figure 1):

- A mobile part, shown in Figure 2, composed of a coarse positioning device using a piezomotor from PiezoMotor ${ }^{\mathscr{Q}}$ and a piezoelectric bending cantilever for fine positioning. This cantilever is the first finger of the gripper.

- A fixed part, linked to the main support which constitutes the second finger of the gripper. 


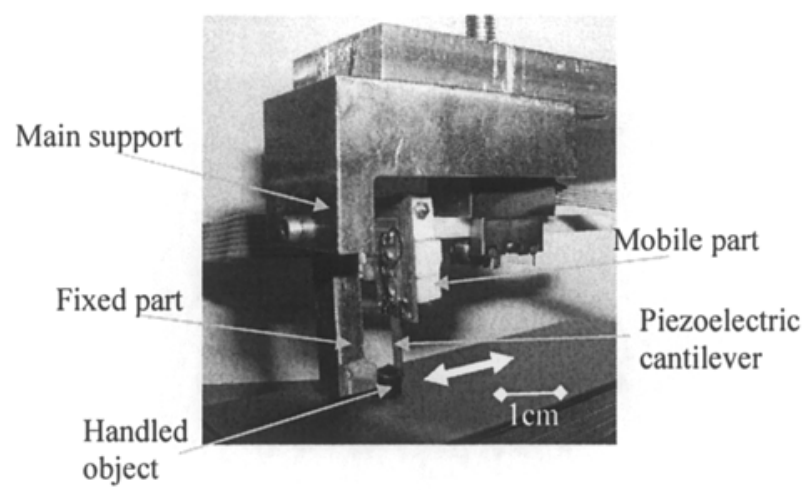

Fig.1. Structure of the microgripper

\section{Force measurement}

To perform force measurement, a strain gauge (length: $1.27 \mathrm{~mm}$, width: $0.38 \mathrm{~mm}$ ) is glued on every side of the piezoelectric cantilever (see Figure 2) which is bonded on a PCB. The force measurement depends on the gauge properties and the physical characteristics and dimensions of the cantilever. Other principles of force measurement are also possible [2] [3] [4].

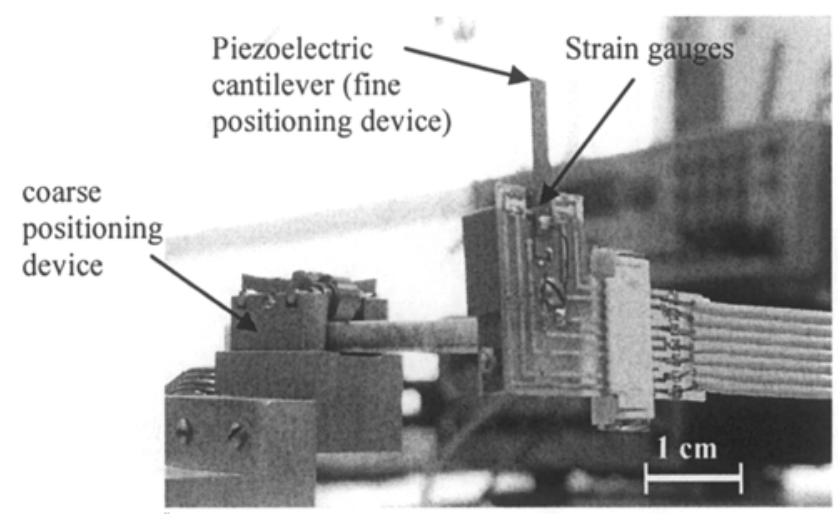

Fig. 2. The mobile part equipped with strain gauges

\subsection{Principle of the cantilever piezoelectric beam}

Piezoelectricity is the property of some materials (crystals and ceramics) to generate electrical charges in response to a mechanical stress. This is the direct piezoelec- 
tric effect. The reverse piezoelectric effect is the production of shape change when an electrical field is applied. The cantilever is composed of two PZT layers (see figure 3) because this structure allows larger deflexion than a cantilever with only one PZT layer. The dimensions of the cantilever are: length: $16 \mathrm{~mm}$, width: $2 \mathrm{~mm}$ and height: $0.5 \mathrm{~mm}$. The bending cantilever response is studied and given in [5]. This bending cantilever is considered as two inputs/one output system. The inputs are the force $F$ applied at the tip of cantilever and the voltage $V$ applied across the electrodes. The output is the deflexion $\delta$ of the cantilever tip. These signals are linked by the following equation:

$$
\delta(s)=\underbrace{\frac{\alpha}{a s^{2}+b s+1}}_{\text {dynamic behaviour }} F(s)+\underbrace{\frac{\beta}{a s^{2}+b s+1}}_{\text {dynamic behaviour }} V(s)
$$

where:

$\mathrm{s}$ is the Laplace variable and $\alpha, \beta, a, b$ and $c$ are calculated from the physical properties and dimensions of the cantilever.

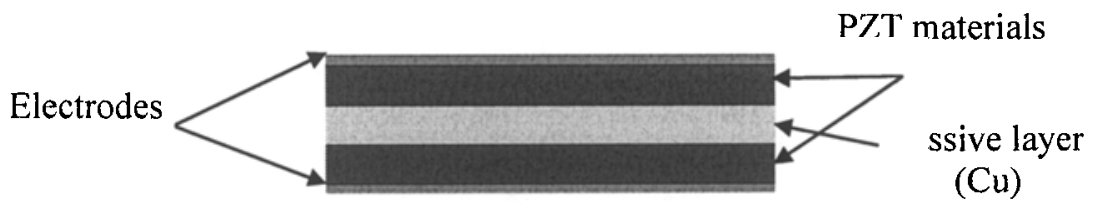

Fig. 3. Bending cantilever structure

\subsection{Calibration}

For the measurement, the strain gauges are integrated in a Wheatstone bridge. The output signal is amplified (gain 51.3) and filtered with a low pass filter with a cutoff frequency of $247 \mathrm{~Hz}$, in order to obtain a suitable range for the feedback signal. Step identification was done using $\mathrm{dSPACE}^{(3)}$ board and a laser sensor from KEYENCE $^{\circledR}$ (resolution: 10nm). The Wheatstone bridge associated with the conditioner (filter + amplifier) provides $50.75 \mathrm{mV} / \mathrm{mN}$ sensitivity.

The response is:

$$
\delta(s)=\frac{1.22}{3.43 * 10^{-8} s^{2}+9.26 * 10^{-6} s+1} V(s)+\frac{1.231}{3.43 * 10^{-8} s^{2}+9.26 * 10^{-6} s+1} F(s)
$$




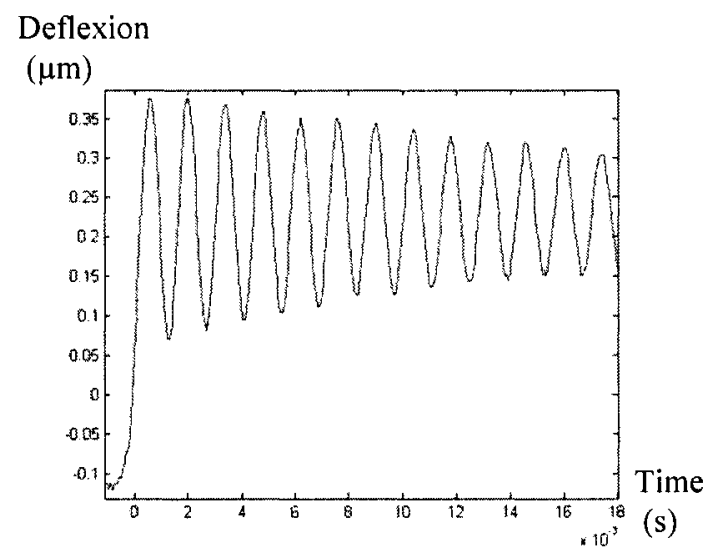

Fig. 4. The cantilever step response when applying the input voltage

The movement of the piezomotor is based on stick-slip principle and has its own controller box PDA 3.1 from PiezoMotor ${ }^{\circledR}$. The piezomotor provides $10 \mathrm{~nm}$ resolution in bending mode and $7.3 \mathrm{~N}$ holding force. The velocity of piezomotor was determined by using laser sensor. The measurements show the non linearity of the velocity according to control voltage and the non symmetrical behaviour (see Figure $5)$.

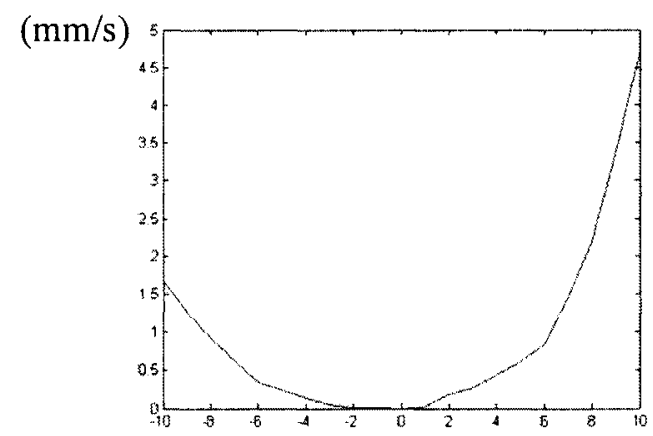

Fig. 5. Velocity of the piezomotor VS control voltage

\section{Force controlled gripping tasks}

The control of gripping force has many applications. During the grasping task, the force control has to prevent the release of the object without exceeding force limit. The microgripper is able to handle small components in a wide range of sizes (from $500 \mu \mathrm{m}$ to few $\mathrm{mm}$ ). The applied force on the object is limited to $81 \mathrm{mN}$ for staying 
in the linear range.

The accuracy of force measurement depends on the output signal noise. In steady state, figure 6 shows the measured noise.

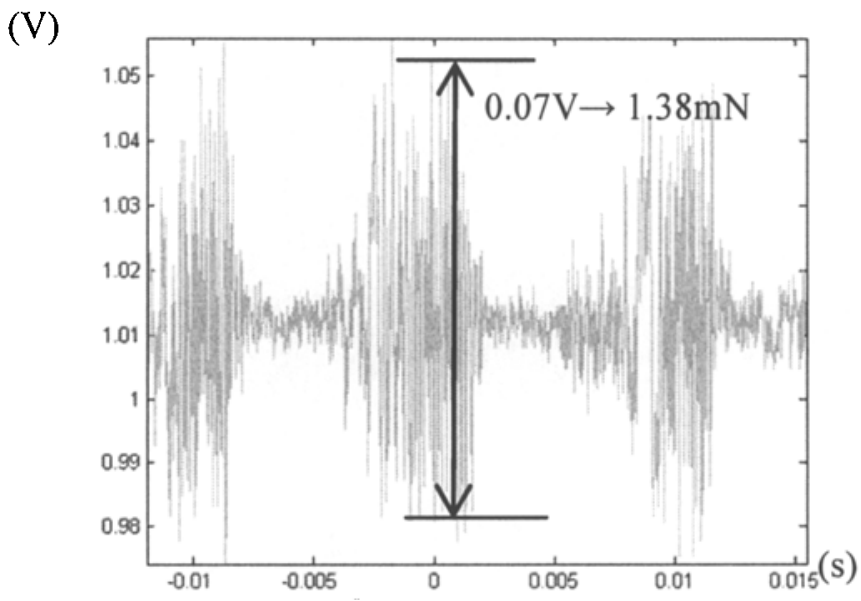

Fig. 6. Noise in steady state

\section{Microgripper adapted to EUPASS Control Structure}

The system has been adapted to the EUPASS Control Structure. For this, Beckhoff provided to EUPASS members the EUPASS Template Library. EUPASS Template Library is composed of the library of functions and function blocs and, the template program (written in Structured Text - IEC 61131-3) which is used to build up the customer specific modules.

In this application, the bending cantilever is used only for force measurement and the grasping is done by the piezomotor movement. The cantilever associated with strain gauges is used as force sensor. The EUPASS Control uses Beckhoff Embedded PC. This control structure supports OMAC (Open Modular Automation Control) PackML guidelines (State, Mode and Communication). The Beckhoff Embedded PC (CX1020) is used for controlling the microgripper in agreement with the EUPASS hardware specification. In combination with TwinCAT (The Window Control and Automation Technology), it works like a PLC (Programmable Logic Controller).

The CX1020 is associated with an analog input which converts the feedback signal and an analog output which controls the piezomotor. Input/Output work with 16bits resolution. 


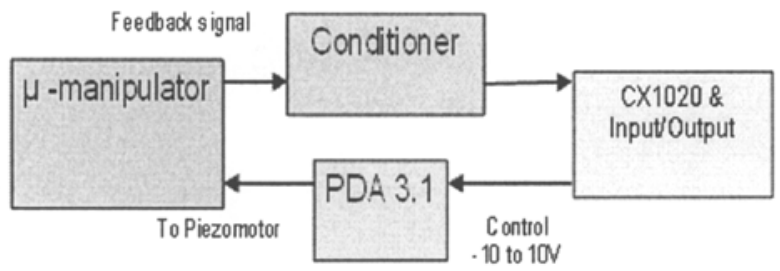

Fig. 7. Block Diagram of the system

Thanks to the structure of the microgripper (see Figure 1), the movement is divided into three steps. In the first step, the microgripper leaves its initial position to go near the object. This displacement is done with a constant speed which is calculated by calibration results. In the second step, the force control is processed by the function bloc PID during a defined time. This function is available in the library ("Tc.utilities.lib"). In the last step, the mobile part goes back to its initial position and grasp work is finished.

This function bloc uses the continuous PID which is discretized with the trapezoidal rule.

$$
G(s)=k_{p}\left(1+\frac{1}{T_{n} s}+\frac{1+T_{v} s}{T_{d} s}\right)
$$

$k_{p}:$ Proportionnal gain, $T_{n}:$ Integral gain,

$T_{v}:$ Derivative gain, and $T_{d}:$ Attenuation Derivative gain

Figure 8 shows the force during the grasping task.

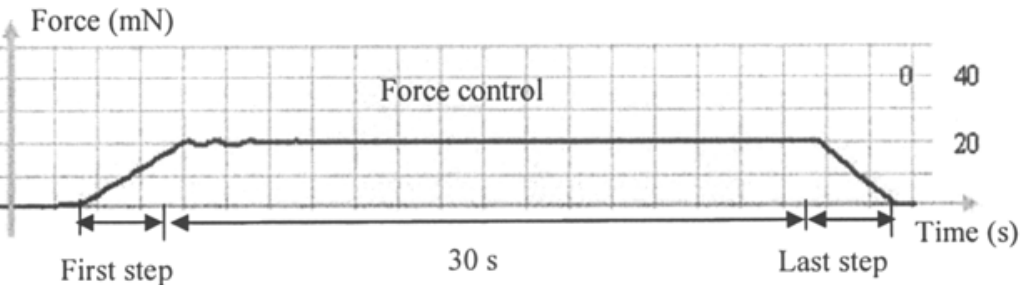

Fig. 8. Stable force control

PID's parameter: $k_{p}: 0.045, T_{n}: 0.035, T_{v}: 0$ et $T_{d}: 1$ 
The different steps of the movement are integrated in "Execute" state according to the EUPASS Template Library. The different steps of the movement correspond to a "Substate". During the test, the microgripper works in Automatic mode and its state switches between "Execute" and "IDLE".

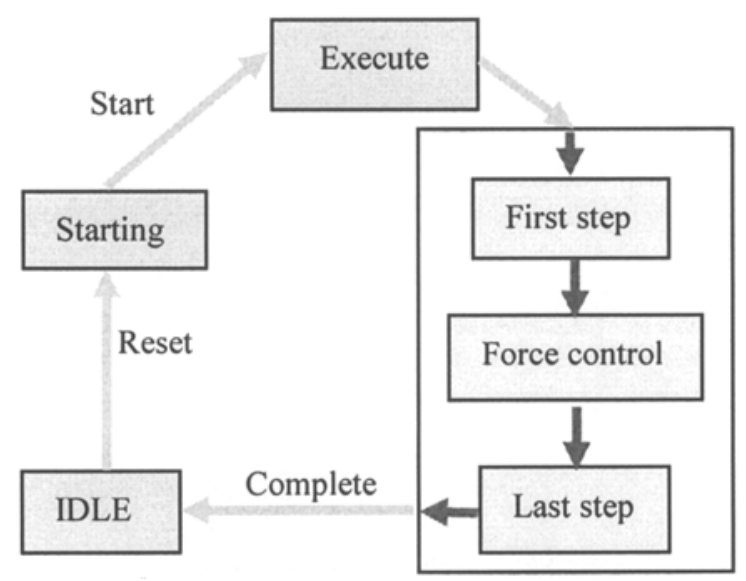

Fig. 9. Bloc diagram of the sequential state and substate

This microgripper can be integrated in an assembly cell or assembly line and cooperate with other EUPASS modules (Pick \& Place, Gluing, etc.).The modules are able to communicate using distributed Master-Master Ethernet network and various topologies would be possible. The synchronisation task is done with a Publisher/Subscriber data exchange via real-time network among the modules.

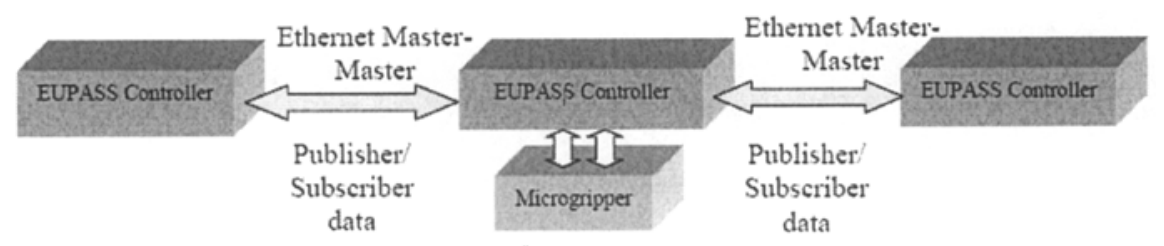

Fig. 10. EUPASS architecture

\section{Conclusion}

In this paper, we have presented a piezoelectrically actuated microgripper which performs force measurement using bimorph cantilever and strain gauges. The force measurement avoids damaging or destroying the manipulated micro-object (from $500 \mu \mathrm{m}$ to few $\mathrm{mm}$ ). The maximum measurable force is $81 \mathrm{mN}$ and the force measurement error is $1.38 \mathrm{mN}$. 
242 A Low Cost Coarse/Fine Piezoelectrically Actuated Microgripper with Force Measurement Adapted to the EUPASS Control Structure

This microgripper is adapted to the EUPASS Control Structure which ensures the standards to integrate an assembly cell or assembly line. This microgripper is able to cooperate with other modules in order to perform micro-assembly tasks.

In a future work, the bending cantilever will be used as an actuator to improve the dynamic characteristics of the gripper and the piezomotor for large displacements.

\section{Acknowledgments}

This work has been done in EUPASS project, supported by the public funding of the European Commission.

\section{References}

1. Qiao C., Yassine H., Philippe L. (UFC), Gebhard M. (Festo), A Low Cost Coarse/Fine Piezoelectrically Actuated Microgripper with Force Measurement, EUSPEN May (2007)

2. Markus Kemper. Development of a Tactile Low-Cost Microgripper with integrated Force Sensor. International Conference on Control Applications Taipei, Taiwan, September 2-4, 2004.

3. Lu, Z., Chen, Peter C. Y., and Lin, W., Force sensing and control in micromanipulation. IEEE Transactionson Systems, Man, and Cybernetics, Part C. September 2005.

4. Eisinberg A., Menciassi A., Micera S., Campolo D., Carrozza M.C., Dario P.," PI force control of a microgripper for assembling biomedical microdevices", IEE Proc. - Circuits Devices Syst., August 2001.

5. Joël Agnus, Contribution to the micromanipulation: studies, realization and command of a piezoelectric grip, November 2003. 